\title{
REVISIÓN DE LA ATENUACIÓN COMO EXPRESIÓN DE LA COMPETENCIA PRAGMÁTICA
}

REVISION OF MITIGATION AS AN EXPRESSION OF PRAGMATIC COMPETENCE

\section{Àngela Magraner Mifsud}

Universitat de València

Resumen

En este trabajo se evalúa la competencia pragmática de estudiantes adolescentes (15-18 años) a partir del análisis de un corpus de muestras escritas que recogen diversas situaciones comunicativas propuestas a los jóvenes. Para llevar a cabo dicha evaluación se ha diseñado un sistema basado en el mayor o menor uso de la estrategia pragmática de la atenuación en el que se establecen tres niveles de adecuación pragmática: adecuado, inadecuado por defecto e inadecuado por exceso. Este sistema nos ha permitido juzgar la competencia pragmática, $\mathrm{y}$, por tanto, también comunicativa de los estudiantes. Los resultados del estudio dan cuenta, por un lado, de la idoneidad de la atenuación como herramienta indicadora de la competencia pragmática en los estudiantes, y por otro lado, muestra las carencias y puntos débiles de los estudiantes de secundaria en el desarrollo de este fenómeno pragmático. A partir de estos resultados, se ha diseñado una propuesta didáctica para el desarrollo de las estrategias de atenuación y su uso adecuado en estudiantes de este nivel de estudios. En concreto, se pretende promover su competencia pragmática en cuanto a la producción e interpretación de enunciados en contextos específicos.

PALABRAS CLAVE: atenuación, competencia comunicativa, competencia pragmática, fuerza ilocutiva, situación comunicativa
Abstract

In this paper, the pragmatic competence of adolescent students (15-18 years old) is evaluated. The study in issue focuses on the analysis of a corpus formed by written samples that include various communicative situations proposed to teenagers. In order to carry out this evaluation, a system based on the greater or lesser use of the pragmatic strategy of mitigation has been designed with the establishment of three levels of pragmatic adequacy: adequate, inadequate by default and inadequate by excess. This system enables us to judge the pragmatic and, therefore, also the communicative competence of the students. The results of the study state, on the one hand, the suitability of the mitigation as an indicator of the pragmatic competence among students and, on the other hand, the deficiencies and weak points of secondary education students in the development of this pragmatic phenomenon. Based on these results, a didactic proposal has been designed for the development of mitigation strategies and their appropriate use in students attending the level under consideration. It specifically aims to promote its pragmatic competence in terms of the production and interpretation of statements in specific contexts.

KEY WORDS: mitigation, communicative competence, pragmatic competence, illocutionary force, communicative situation. 


\section{INTRODUCCIÓN}

Como es sabido, Bachman (1990) reconoce la competencia pragmática como una de las dos subcompetencias principales de la competencia comunicativa. Sin embargo, el sistema de enseñanza del español en secundaria se centra casi de forma exclusiva en la competencia organizativa (que incluye, la gramatical y la textual, Bachman, 1990), olvidándose de la parte pragmática de la lengua, sin la cual, los hablantes no lograríamos ser eficientes en nuestros intercambios comunicativos diarios. El presente trabajo se suma a aquellos que reivindican la mayor atención a la competencia pragmática en los cursos de lengua en el sistema educativo de la secundaria (Lomas, 1994 y 1997; Lomas, Osoro y Tusón, 1993; Fernández Vallejo, 1998; Bulnes Jiménez, 1993): enseñar a comunicarse en una lengua no puede reducirse a ser competentes gramatical y textualmente, sino que resulta imprescindible enseñar también mecanismos pragmáticos que nos ayuden, por una parte, a conseguir nuestro propósito comunicativo (competencia ilocutiva) y, por otra, a reconocer las características del contexto de uso de la lengua para producir enunciados o textos adecuados a ella (competencia sociolingüística).

En concreto, nos centramos en un mecanismo de naturaleza pragmática, la atenuación lingüística, ya que, como veremos, su uso puede ser determinante para conseguir el éxito comunicativo. Se ha evaluado la adecuación en el uso de este mecanismo en estudiantes de $4^{\circ}$ de la ESO. Para ello, se ha llevado a cabo un estudio empírico en el que se ha recogido un corpus escrito de 88 actividades en las que se presentan situaciones comunicativas concretas que los estudiantes tienen que resolver. Para efectuar la evaluación de la competencia pragmática se ha diseñado un sistema basado en el mayor o menor uso de la atenuación en el que se establecen tres niveles de adecuación pragmática: adecuado, inadecuado por defecto e inadecuado por exceso (basado parcialmente en Secchi, 2017).

Por último, como el objetivo final del estudio es mejorar la realidad educativa del aula, después de haberla analizado y atendiendo a las principales carencias detectadas en el análisis, se ha diseñado una propuesta didáctica dirigida al alumnado de $4^{\circ}$ de la ESO, basada en la estrategia de la atenuación, a través de la que se pretende desarrollar su competencia pragmática en cuanto a interpretación de enunciados y su producción en contextos concretos. Para diseñar y elaborar las distintas actividades que conforman la propuesta, se ha seguido un enfoque comunicativo en el que se ha provocado y favorecido en los estudiantes la necesidad de comunicarse (tanto oralmente como por escrito) para poder resolver las situaciones y problemas planteados. En concreto, gran parte de la propuesta didáctica sigue el enfoque por tareas.

\section{MARCO TEÓRICO}

Aunque «las competencias han estado presentes a lo largo del siglo XX en numerosos programas formativos y han significado un campo de investigación intensa en el campo de la enseñanza» (Medina, 2009: 12), no es hasta el año 2000 cuando, en el Consejo Europeo de Lisboa, se acuerda la necesidad de un marco europeo que, una vez identificadas, defina las nuevas destrezas básicas que se han de adquirir, determine la forma de integrarlas en el currículo y posibilite un aprendizaje a lo largo de la vida. Tal acuerdo se pone en marcha con el Proyecto DeSeCo (Definición y Selección de Competencias, 2002), que se realiza en doce países y está vinculado al Proyecto INES (International Indicator for Education Systems). Estos proyectos definen competencia como la capacidad de responder a demandas complejas para llevar a cabo tareas diversas de forma adecuada, constituyendo 
de esta forma un saber aplicado, contextualizado y, por lo tanto, susceptible de adecuarse a una diversidad de situaciones y ámbitos que puede clasificarse en tres categorías: la actuación de forma autónoma, la utilización interactiva de herramientas y el funcionamiento en grupos socialmente heterogéneos (González et alii, 2011: 39).

Así, el aprendizaje por competencias constituye un nuevo enfoque educativo que «tiene como objetivo prioritario ofrecer respuestas a algunos de los grandes desafíos que determinan nuestro momento histórico', concibiendo la educación obligatoria como base para el aprendizaje permanente, para un constante "estar al día"» (González et alii, 2011: 42). En España es la Ley Orgánica de Educación, 2/2006 de 3 de mayo, la que introduce el concepto de competencia básica en los currículos y la que establece las ocho competencias básicas que deben desarrollarse en la enseñanza obligatoria española. En este trabajo nos centraremos en la competencia en comunicación lingüística. Dado que las personas van construyéndose como tales en la interacción con otras y que aprender es básicamente una actividad social, "saber comunicarse se convierte en la competencia clave en el acceso a la información, en la construcción del conocimiento y, por tanto, en la formación de alumnos competentes» (González et alii, 2011: 61-62).

Dominar la competencia comunicativa implica producir, expresar e interpretar diferentes tipos de discurso acordes a la situación comunicativa en diferentes contextos sociales y culturales, gracias al conocimiento y a la aplicación efectiva de las reglas de funcionamiento del sistema de la lengua, para lo que es necesario las habilidades tanto lingüísticas como no lingüísticas (Medina, 2009: 104). De entre los diversos modelos de competencia comunicativa propuestos, en este trabajo se sigue el propuesto por Bachman (1990), por tratarse de uno de los primeros en incluir la competencia pragmática. En algunos modelos anteriores, si bien no se hace referencia explícita a la competencia pragmática, su contenido puede considerarse integrado en otros componentes de la competencia comunicativa (Hymes, 1972; Canale y Swain 1980; Canale, 1983; Van Ek, 1986). Según Bachman, la competencia en la lengua (competencia comunicativa) se estructura del siguiente modo:

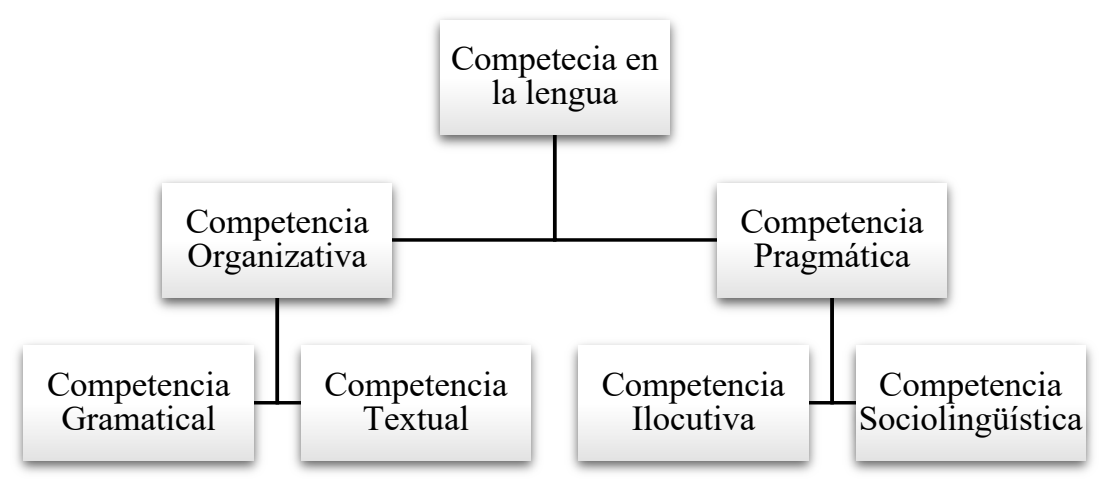

Figura 1. Modelo de competencia comunicativa de Bachman (1990)

Se entiende por competencia pragmática «la habilidad de transmitir y comprender el intento comunicativo por medio de la ejecución e interpretación de los actos de habla y las funciones lingüísticas» (Celce-Murcia, Dornyei y Thurrell, 1995: 9). Bialystok (1993: 43) la

\footnotetext{
${ }_{1}^{1}$ Nos referimos a retos como el de la alfabetización digital, el multilingüismo, el flujo de enormes cantidades de información, la sostenibilidad medioambiental o la globalización, entre otros. 
describe del siguiente modo:

\begin{abstract}
Pragmatic competence entails a variety of abilities concerned with the use and interpretation of language in contexts. It includes speakers' ability to use language for different purposes -to request, to instruct, to effect change-. It includes listeners' ability to get past the language and understand the speaker's real intentions, especially when these intentions are not directly conveyed in the forms -indirect requests, irony and sarcasm are some examples-.
\end{abstract}

Centrándonos en el modelo de competencia pragmática propuesto por Bachman, esta está formada por:

a. La competencia ilocutiva, que se refiere a la relación entre los enunciados y actos o funciones que los hablantes o escritores intentan realizar a través de estos enunciados. Un enunciado puede ser correcto gramaticalmente pero inadecuado por falta de competencia ilocutiva:

\title{
A: Tienes dos bolis azules? \\ B: Sí (pero no le presta uno)
}

Aquí, la intención del hablante A era la de que su compañero le dejara uno de los dos bolis azules que tenía, pero B entiende la pregunta de $A$ en sentido literal y no con la función de petición que ha querido darle. Por tanto, se trata de un caso en el que la competencia ilocutiva falla.

b. La competencia sociolingüística, que hace referencia a las características del contexto de uso de la lengua que determinan qué enunciados son apropiados y cuáles no en determinadas situaciones comunicativas.

Así, es conveniente pensar en un concepto de competencia comunicativa que resalte la importancia de los elementos pragmáticos en la comunicación y su posterior efecto en la didáctica de la lengua, ya que, para enseñar a comunicar en una lengua es imprescindible enseñar también este componente de la competencia comunicativa. En este sentido, nos proponemos fomentar el desarrollo de la competencia pragmática en los estudiantes de secundaria, y en concreto, demostrar que la estrategia de la atenuación constituye una buena forma de mejorar la competencia y la adecuación pragmática.

En cuanto al fenómeno de la atenuación, seguimos la propuesta de caracterización de Briz y Albelda (2013: 292): «La atenuación es una categoría pragmática en tanto mecanismo estratégico y táctico (por tanto, intencional), que tiene que ver con la efectividad y la eficacia del discurso, con el logro de los fines en la interacción, además de tratarse de una función solo determinable contextualmente».

Se considera que es una estrategia, y por tanto, un mecanismo que contribuye a la negociación y al logro del acuerdo entre los interlocutores. Es, en este sentido también, un mecanismo retórico para convencer, lograr un beneficio, persuadir y, a la vez, para cuidar las relaciones interpersonales y sociales o evitar que estas se deterioren o sufran algún tipo de daño. Así, el uso adecuado de esta estrategia pragmática puede contribuir de forma muy significativa a que un determinado mensaje o enunciado sea pragmáticamente adecuado o no. Fijémonos en el siguiente ejemplo (1). Se trata de una actividad extraída 
del corpus $^{2}$, realizada por una alumna de $4^{\circ}$ de la ESO, en la que vemos diversos mecanismos atenuantes resaltados tipográficamente en cursiva:

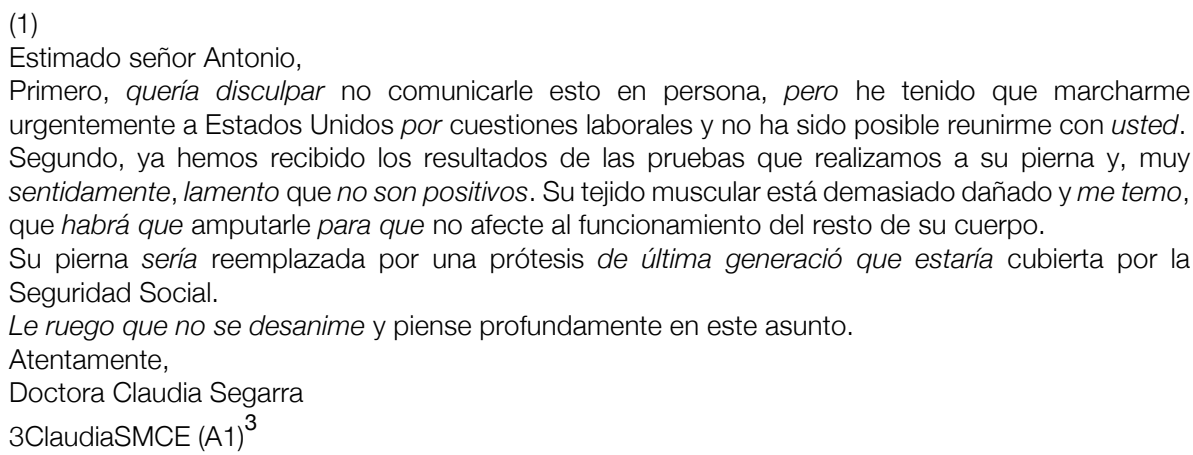

Una prueba que manifiesta la relevancia de esta estrategia pragmática en el logro de una competencia pragmática eficiente consiste en suprimir los mecanismos atenuantes del ejemplo anterior, como se puede ver en (1') a continuación:

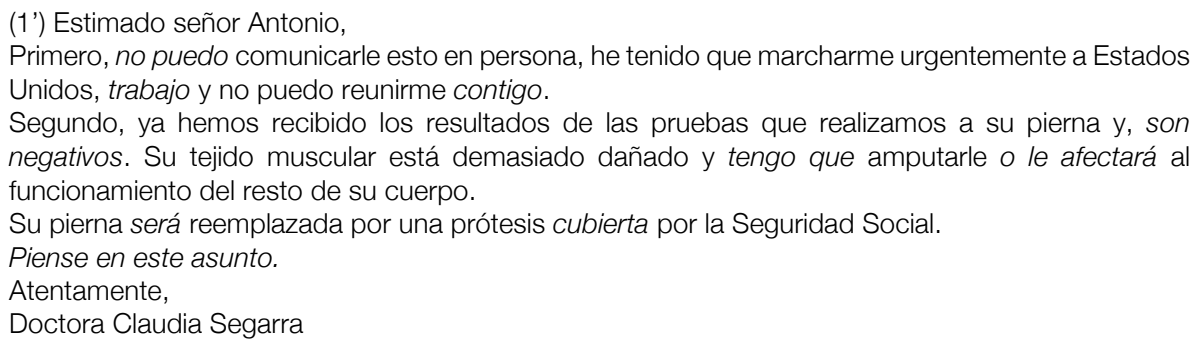

En (1') se aprecia un mensaje más directo, que únicamente incorpora el contenido informativo que el emisor quiere transmitirle a su interlocutor. Si bien se logra el traspaso de información, seguramente la eficiencia comunicativa de (1') es muy pobre. Así, el dominio de las subcompetencias gramaticales y la corrección no son suficientes para la comunicación. Los mecanismos pragmáticos y, en concreto, la atenuación ayuda, por una parte a conseguir el propósito comunicativo o competencia ilocutiva (en este caso, transmitir una mala noticia sin dañar los sentimientos o la imagen del paciente) y, por otra, a reconocer las características del contexto de uso de la lengua para poder adecuarnos a él. Por tanto, los mecanismos pragmáticos en general y la atenuación en particular, constituyen un factor decisivo para considerar un determinado acto comunicativo como adecuado o inadecuado pragmáticamente.

\section{Metodología}

Para la adquisición de la competencia pragmática y, por tanto, para poder evaluarla en los estudiantes, es fundamental hacerlo juzgando cada uso lingüístico de acuerdo con su contexto y situación comunicativa. En la enseñanza de esta competencia se ha de ayudar al alumno a que antes de realizar la actividad o de emprender cualquier acto comunicativo, tenga presente los elementos de la situación comunicativa. De acuerdo con Briz (1998), son los cuatro siguientes: el marco físico en el que se desarrollará la comunicación (familiar/cotidiano o no familiar/cotidiano), la relación de poder/ jerarquía entre los

\footnotetext{
2 Todas las actividades citadas están transcritas literalmente tal y como las han realizado los estudiantes, incluyendo faltas de ortografía, de redacción, de puntuación y de estilo.

${ }^{3}$ Cada uno de los ejercicios está etiquetado de esta forma, cuyos caracteres representan lo siguiente: 3 , tercera alumna del grupo; Claudia, el nombre; SM, las iniciales de sus apellidos; C, del grupo C; E de la nacionalidad, española; y (A1), de actividad 1. 
interlocutores (igualdad o desigualdad funcional o social), el grado de proximidad o el conocimiento común compartido con el/los interlocutor/es (proximidad o desconocimiento) y el origen geográfico y cultural del hablante (cultura de acercamiento o de distanciamiento). Como explica Briz (1998: 25): «El contexto de comunicación regula y marca de algún modo las conductas lingüísticas y extralingüísticas de los hablantes, los cuales suelen esforzarse en acomodar en mayor o menor grado sus actos diarios de comunicación a la situación precisa en que tienen lugar».

Esta inadecuación pragmática puede resolverse y corregirse de forma notoria a través del uso de la estrategia de la atenuación, ya que esta es una de las múltiples categorías que afectan a la competencia pragmática, pudiendo ayudar a evitar estos desajustes comunicativos y de conducta. Así, considerar los factores de la situación comunicativa nos permite poder adecuarnos a ellos y ser más efectivos al comunicarnos. En este sentido, los mecanismos de atenuación son procedimientos lingüísticos que regulan la interacción entre los hablantes y actúan en el nivel interpersonal y social de la comunicación. Como se ha señalado, facilitan la relación entre los hablantes, por lo que dominar su uso, facilita la comunicación humana.

\subsection{Sistema de evaluación de la competencia pragmática}

Basándonos en un estudio previo centrado en el uso de la atenuación pragmática en las muestras del presente corpus (Magraner, en prensa), hemos evaluado la adecuación pragmática en cada uno de los textos. Así, siguiendo el modelo propuesto por Secchi (2017), se ha diseñado un sistema de evaluación de la competencia pragmática basado en el mayor o menor uso de la atenuación en el que se han considerado tres niveles de adecuación pragmática:

1) Adecuado: el/la alumno/a se adecúa perfectamente a la situación comunicativa concreta que se le propone, en tanto que ha empleado las estrategias de atenuación apropiadas y necesarias.

2) Inadecuado por defecto: el/la alumno/a no se adecúa a la situación comunicativa concreta que se le propone, en tanto que no ha empleado (suficientes) estrategias de atenuación.

3) Inadecuado por exceso: el/la alumno/a no se adecúa a la situación comunicativa concreta que se le propone, en tanto que ha empleado demasiadas estrategias de atenuación (overpoliteness).

Así pues, hemos considerado inadecuadas pragmáticamente todas aquellas actividades en las que el alumno no utiliza (suficientes) estrategias de atenuación para matizar o suavizar el contenido significativo de su mensaje. Asimismo, también hemos creído oportuno evaluar como inadecuadas pragmáticamente todas aquellas actividades en las que el alumno emplea demasiadas estrategias de atenuación para la situación comunicativa que se le ha presentado, ya que los comportamientos hipercorteses, conocidos como overpoliteness, también son perjudiciales, pues una cortesía exagerada puede mostrar una aplicación pragmática fallida (Bernal, 2007: 76)4.

\footnotetext{
${ }_{4}^{4}$ Bravo (en Albelda y Barros, 2013: 82-83) define la cortesía comunicativa como la «actividad comunicativa que considera el beneficio para la imagen del otro y que, consecuentemente, tiene efectos positivos para el desarrollo armónico de la comunicación y para el equilibrio de las relaciones interpersonales. Responde a normas y a códigos sociales que se suponen en conocimiento de los hablantes». 
Revisión de la atenuación como expresión de la competencia pragmática | À. Magraner

Estos tres niveles de adecuación pragmática se basan en los diferentes tipos de relational work establecidos por Locher y Watts (2005: 11-12):

\begin{abstract}
In terms of individual participants' perceptions of verbal interaction, which is oriented to the norms established in previous interactions, a great deal of the relational work carried out will be of an unmarked nature and will go largely unnoticed (i. e., it will be politic/appropriate, column 2). Marked behavior, conversely, can be noticed in three ways. It will be perceived as negative if it is judged as impolite/non-politic/ inappropriate (column 1), or as over-polite/non-politic/inappropriate (column 4). We hypothesize that addressees' affective reactions to over polite behavior will be roughly similar to their reactions to impolite behavior. Positively marked behavior (column 3) will coincide with its being perceived as polite/politic/appropriate. In other words, polite behavior is always politic while politic behavior can also be non-polite.
\end{abstract}

Como se aprecia en la Tabla 1 de abajo, las correspondencias entre los tipos de trabajo relacional de Locher y Watts (para el estudio de la cortesía verbal) y los tres niveles aquí considerados es la siguiente: el nivel 1 (adecuado) del presente sistema evaluativo se correlaciona con el tercer tipo de relational work (conducta marcada positivamente); el nivel 2 (inadecuado por defecto) se corresponde con el primer (conducta marcada negativamente por descortesía) y el segundo tipo (conducta no marcada, neutra) de relational work5; el nivel 3 (inadecuado por exceso) equivale al tercer tipo de relational work (conducta marcada negativamente por sobrecortesía):

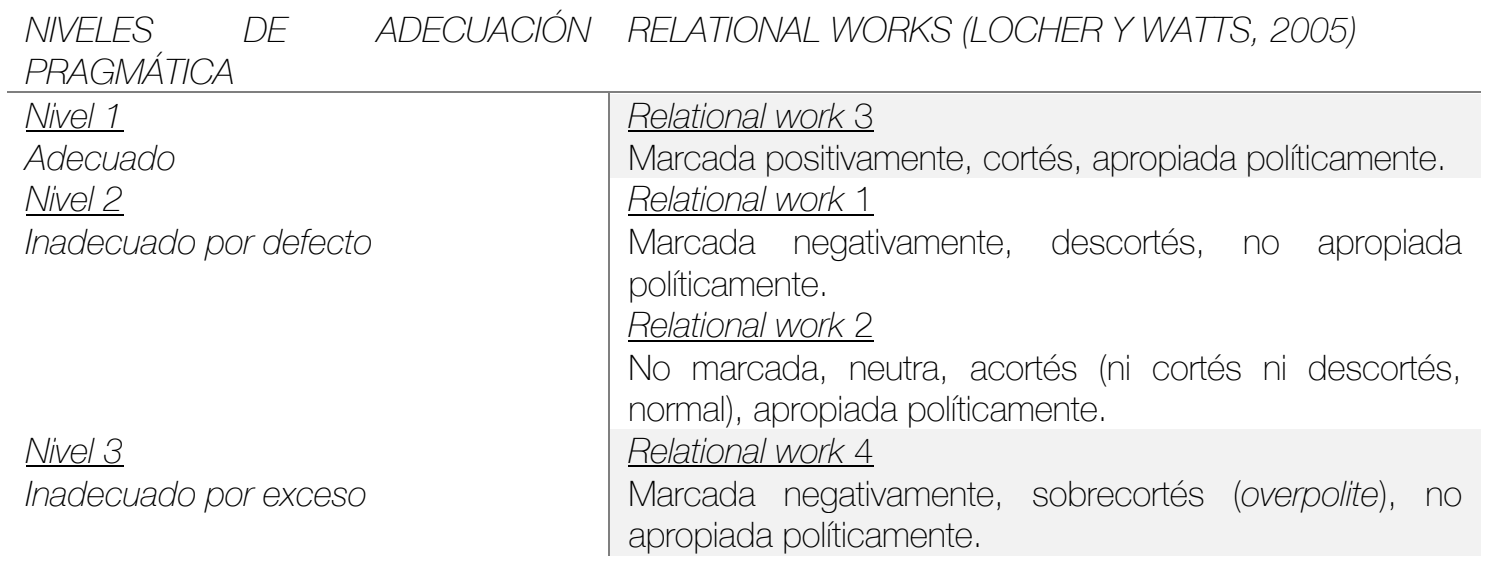

Tabla 1. Relación entre los tipos de relational work de Locher y Watts (2005) y el sistema de evaluación de la adecuación pragmática

Así pues, hemos analizado todas las muestras del corpus de acuerdo con este sistema evaluativo con el objetivo de ver si los estudiantes tienen desarrollada la competencia pragmática y si son capaces de adecuarse a las situaciones comunicativas que se les presentan. Además, también se han intentado suplir las carencias observadas en el análisis del corpus a través del diseño de una propuesta didáctica en la que se desarrolle la competencia pragmática y comunicativa gracias al uso de mecanismos atenuantes.

\title{
3.2 Actividades de los estudiantes y corpus de análisis
}

El corpus de análisis se ha compilado a partir de la petición a los estudiantes de $4^{\circ}$ de la ESO de la compleción de dos tipos de actividades. Se les presenta en cada actividad una situación comunicativa muy concreta, con todo el detalle de la contextualización, como se

\footnotetext{
${ }^{5}$ Se ha considerado que el segundo tipo de relational work se situaría dentro de nuestro sistema en el nivel 2 (inadecuado por defecto) porque las situaciones planteadas en las actividades requieren ser atenuadas, y aquella que no esté marcada y sea acortés, aunque no sea inapropiada, puede dañar la imagen del interlocutor, pues se trata de situaciones delicadas que requieren ser marcadas positivamente. 
muestra abajo en las figuras 1 y 2 . Obsérvese cómo las propias instrucciones de las actividades se conciben desde un enfoque comunicativo, en tanto que se detallan los cuatro rasgos de la situación comunicativa particular en la que han de desarrollar el intercambio comunicativo. Son, además, situaciones reales que se presentan en la vida cotidiana de los adultos; en el caso de la actividad 2 puede ser algo que ellos mismos hayan experimentado en diversas ocasiones .

En la primera actividad, los alumnos realizan un juego de rol y se ponen en el papel de un doctor que tiene que transmitirle una mala noticia a su paciente:

\section{Actividad 1}

Eres un/a doctor/a que trabaja en el Hospital la Fe de Valencia. Ayer atendiste a Antonio, un hombre que había tenido un accidente laboral que le había dejado la pierna destrozada. Hoy tienes que irte fuera, por cuestiones de trabajo, y no podrás comunicárselo en persona, por lo que decides escribirle un correo electrónico informándole de que los resultados de las pruebas no han sido del todo positivos y que finalmente sera̋ necesario amputarle la pierna si quiere sequir con vida.

Figura 2. Instrucción para la realización de la actividad 1

En la segunda actividad, los estudiantes elaboran un acto de habla de rechazo en el que tienen que decirle a su mejor amigo/a, con el que han quedado para ir al cine, que al final no van a poder ir:

\section{Actividad 2}

Habías quedado con tu mejor amigo/a esta tarde para ir al cine, pero te ha salido un plan mejor y no sabes cómo decírselo. Escríbele un WhatsApp a tu amigo/a justificando el motivo por el que finalmente no podrás ir.

Figura 3. Instrucción para la realización de la actividad 2

Como bien indican González y García (2017: 189):

\footnotetext{
El Análisis de la Conversación (AC) ha categorizado el rechazo como una respuesta despreferida dentro de los pares adyacentes en los que el primer miembro del par establece la relevancia de la aceptación o el rechazo (i.e., peticiones, invitaciones, etc.). Por definición, las respuestas despreferidas son más complejas y largas que las respuestas preferidas [...] por la aparición de mecanismos de indirección, tales como la tendencia a retardar la respuesta, la inclusión de excusas o justificaciones que acompañan al rechazo, y la atenuación interna del rechazo en sí.
}

Como se aprecia, las actividades plantean dos situaciones comunicativas distintas, una transaccional y formal y la otra interpersonal e informal; sin embargo, ambas requieren el uso de los mecanismos lingüísticos de la atenuación: la actividad 1 por presentar una situación comunicativa formal en la que se transmite una noticia delicada entre dos interlocutores que no se conocían previamente, y la actividad 2 por constituir un acto de habla de rechazo. Además, es importante tener en cuenta que, tanto en la actividad 1 como en la 2, se proponen situaciones comunicativas en las que se puede dañar fácilmente la imagen de los interlocutores, siendo la atenuación un mecanismo que puede ayudarnos a prevenir el daño o la intromisión en el territorio de la otra persona. Por tanto, el uso de la Normas (ISSN: 2174-7245) | 
atenuación que hagan los estudiantes ha de ser equilibrado y armónico, es decir, el adecuado a las situaciones propuestas, ya que, tanto los comportamientos hipercorteses (overpoliteness) como los insuficientes en el recurso a este fenómeno, serán inadecuados.

Por otro lado, las características de los informantes y del corpus son las siguientes. Las muestras se obtuvieron durante el segundo trimestre (enero-marzo) del curso escolar 2017-2018, en el IES Districte Marítim de la ciudad de Valencia. Un total de 44 alumnos de $4^{\circ}$ de la ESO pertenecientes a tres grupos distintos realizaron las dos actividades explicadas: 21 alumnos del grupo C, 10 del grupo E y 13 del grupo F. A estos alumnos, se les requería, además, los siguientes datos: nombre, apellidos, fecha de nacimiento, curso, grupo, lengua materna y nacionalidad6. Esto nos han permitido establecer distintas variables como el sexo, la edad, la lengua materna o la nacionalidad, que nos han ayudado considerablemente a entender las actividades de cada alumno y a evaluarlas:

\begin{tabular}{|c|c|c|}
\hline \multicolumn{3}{|l|}{ VARIABLES } \\
\hline \multirow[b]{2}{*}{ Sexo } & Mujer: 23 & \multirow{15}{*}{ Total: 44} \\
\hline & Hombre: 21 & \\
\hline \multirow{3}{*}{ Edad } & 2000 (17-18 años): 5 & \\
\hline & 2001 (16-17 años): 16 & \\
\hline & 2002 (15-16 años): 23 & \\
\hline \multirow{3}{*}{ Lengua materna } & Valenciano: 2 & \\
\hline & Castellano: 39 & \\
\hline & Valenciano/castellano: 3 & \\
\hline \multirow{7}{*}{ Nacionalidad } & Español/a: 36 & \\
\hline & Colombiano/a: 2 & \\
\hline & Ecuatoriano/a: 1 & \\
\hline & Dominicano/a: 1 & \\
\hline & Ecuatoriano/a-Español/a: 2 & \\
\hline & Colombiano/a-Español/a: 1 & \\
\hline & Británico/a-Español/a: 1 & \\
\hline
\end{tabular}

Tabla 2. Variables consideradas en el corpus

En definitiva, se ha trabajado con un corpus escrito en español, formado por 88 actividades realizadas por 44 alumnos alumnos diferentes y con alrededor de 8000 palabras.

\section{RESULTADOS DEL ANÁLISIS}

Veamos ahora los resultados del análisis del corpus en lo relativo a la (in)adecuación pragmática en el uso de la atenuación. Siguiendo el sistema evaluativo presentado en la metodología e integrado por tres niveles de adecuación pragmática, hemos determinado si las actividades eran o no adecuadas pragmáticamente. En la tabla 3 se recogen los porcentajes que representan dentro del corpus cada uno de los niveles de adecuación pragmática:

\footnotetext{
${ }^{6}$ Esto puede ayudar a entender un menor dominio de la competencia lingüística y también de la competencia pragmática, ya que si pertenecen a otros países o culturas, las formas de relacionarse e interactuar también serán diferentes. Normas (ISSN: 2174-7245) | 


\begin{tabular}{|c|c|c|c|}
\hline \multicolumn{2}{|c|}{ NIVEL DE ADECUACIÓN PRAGMÁTICA } & $\begin{array}{l}\text { No ABSOLUTO DE } \\
\text { ACTIVIDADES }\end{array}$ & \multirow{2}{*}{$\begin{array}{l}\text { PORCENTAJE EN EL } \\
\text { CORPUS TOTAL } \\
59,09 \%\end{array}$} \\
\hline 1 & Adecuado & 52 & \\
\hline 2 & Inadecuado por defecto & 31 & $35,22 \%$ \\
\hline 3 & Inadecuado por exceso & 5 & $5,68 \%$ \\
\hline \multicolumn{2}{|c|}{ TOTAL } & 88 & $100 \%$ \\
\hline
\end{tabular}

Tabla 3. Resultados generales de la evaluación de la competencia pragmática

La Tabla 3 muestra cómo en la mayoría de los casos (59,09\%) los alumnos han sabido adecuarse a la situación comunicativa propuesta, haciendo un buen uso de la competencia pragmática, para lo cual se han ayudado y apoyado en el uso de las estrategias de atenuación apropiadas y necesarias en cada caso. Sin embargo, ha de hacerse notar que incluso las actividades que han sido consideradas adecuadas, podrían mejorarse a través del empleo de la atenuación, lo que les permitiría desempeñar un mayor uso retórico de la lengua y asegurarse de que los destinatarios reciben los mensajes con mayor aceptación.

Asimismo, más del 40\% de actividades han sido inadecuadas, ya sea por defecto $(35,22 \%)$ o por exceso (5,68\%). Este resultado pone de manifiesto la necesidad de diseñar una propuesta didáctica basada en la implementación de la atenuación que les permita desarrollar una buena competencia pragmática y ser eficientes comunicativamente.

También resulta informativo observar en cuál de las dos actividades los alumnos se han comportado de manera más adecuada, lo que nos indica qué tipo situaciones comunicativas saben afrontar mejor y cuáles tienes más descuidadas, y, por tanto, cuáles debemos trabajar más en el aula. La tabla 4 recoge los resultados del análisis de la adecuación pragmática de cada una de las actividades de forma comparativa:

ACTIMDAD 1

ACTIVIDAD 2

\begin{tabular}{|c|c|c|c|c|c|}
\hline $\begin{array}{l}\text { Nivel de } \\
\text { adecuación } \\
\text { pragmática }\end{array}$ & $\begin{array}{l}\mathrm{N}^{\mathrm{O}} \\
\text { actividades }\end{array}$ & $\begin{array}{l}\text { Porcentaje en el } \\
\text { corpus analizado }\end{array}$ & $\begin{array}{l}\text { Nivel de } \\
\text { adecuación } \\
\text { pragmática }\end{array}$ & $\begin{array}{l}\mathrm{N}^{\mathrm{O}} \\
\text { actividades }\end{array}$ & $\begin{array}{l}\text { Porcentaje en } \\
\text { el corpus } \\
\text { analizado }\end{array}$ \\
\hline 1. Adecuado & 21 & $47,72 \%$ & 1. Adecuado & 31 & $70,45 \%$ \\
\hline $\begin{array}{l}\text { 2. Inadecuado } \\
\text { por defecto }\end{array}$ & 20 & $45,45 \%$ & $\begin{array}{l}\text { 2. Inadecuado } \\
\text { por defeco }\end{array}$ & 11 & $25 \%$ \\
\hline $\begin{array}{l}\text { 3. Inadecuado } \\
\text { por exceso }\end{array}$ & 3 & $6,81 \%$ & $\begin{array}{l}\text { 3. Inadecuado } \\
\text { por exceso }\end{array}$ & 2 & $4,54 \%$ \\
\hline TOTAL & 44 & $100 \%$ & TOTAL & 44 & $100 \%$ \\
\hline
\end{tabular}

Tabla 4. Resultados de la evaluación de la competencia pragmática: actividad 1 - actividad 2

La Tabla 4 muestra cómo la actividad 2 presenta un grado mucho más alto de adecuación pragmática $(70,45 \%)$ que la actividad 1 (47,72\%). La explicación es sencilla: la situación comunicativa presentada en la actividad 2, además de tener un carácter más informal, es una situación real y habitual para ellos, mientras que no lo son las profesionales, pues aún no se mueven en ese ámbito vital. De ahí que sean más adecuados pragmáticamente a la hora de escribir un WhatsApp que un correo electrónico. En el siguiente ejemplo podemos ver una muestra de un caso en que se ha juzgado como excelente la adecuación pragmática por parte del alumno en la actividad $2^{7}$ :

${ }^{7}$ Se han resaltado tipográficamente en cursiva las tácticas lingüísticas atenuantes empleadas. 
(2) Tio al final no puedo ir, lo siento pero te juro que va enserio, me es imposible, tengo que cuidar de mi hermano que esta con 39 de fiebre y mi madre esta trabajando, si quieres quedamos el viernes y te invito si quieres pero es que hoy es imposible $\theta$... No te piques

En el ejemplo (2) vemos cómo el alumno se adecúa muy bien pragmáticamente no solo por emplear las estrategias de atenuación apropiadas y necesarias para la situación comunicativa concreta que se le ha presentado, sino también por representar a través de dibujos y símbolos (doble tic azul, hora de envío) la actividad en el "ciberespacio" que se le requería, el WhatsApp.

Por su parte, la actividad 1 les ha resultado más compleja, como se aprecia por los resultados, siendo mayor el número de muestras inadecuadas ( $23=52,26 \%$ ) que adecuadas $(21=47,72 \%)$. Esta inadecuación la podemos ver en actividades como la siguiente:

(3) Buenas Antonio, me temo que por temas de trabajo no podré comunicarte una importante decisión que debes tomar... Después de que aquella furgoneta te atropellase, la vena femoral se rompió, esto significa lo siguiente, se está desangrando por dentro con lo que puede causar la muerte, llegados a este punto solo le queda la opción de amputarle la pierna.

Espero que le vaya todo muy bien

Doctor. Esteso

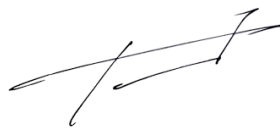

6AdriánABEE (A1)

En esta actividad el alumno no se adecúa por no emplear suficientes estrategias de atenuación para la situación comunicativa concreta que se le ha presentado, ya que no aprecia plenamente que se enfrenta a una situación delicada en la que tiene que dar una mala noticia y que esta requiere ser atenuada. Así, en la mayor parte del texto, el estudiante no demuestra implicación o empatía emocional con la persona a la que se dirige y con el mensaje que transmite. El uso de mecanismos atenuantes como palabras o expresiones cuyo contenido semántico expresa solidaridad y empatía con el interlocutor al que se dirige o como los términos o expresiones más suaves en el contenido significativo, podrían haber suavizado la transmisión de esta información. Por tanto, vemos cómo en esta muestra el alumno no consigue adecuarse pragmáticamente a la situación comunicativa requerida al mostrar una cierta indiferencia hacia el paciente y hacia su enfermedad.

Pero la inadecuación no viene siempre de la mano de la escasez, sino que también puede estar originada por el exceso, como vemos en (4):

(4) Buenos días, estimado Antonio:

Le hablo a estas horas de la mañana para comunicarle algo un poco delicado, me encantaría comunicarselo en persona pero me es un poco difícil por temas 
de trabajo. Bien, es sobre los resultados de sus pruebas, lamento decirle que no han salido del todo bien.

Aunque suene un poco frío decírselo por aquí, es bastante urgente que le amputemos la pierna, ya que lo que le sucede en la piel ha afectado bastante, hasta sus huesos. Por lo que se puede expandir en todo su cuerpo hasta dejarlo sin vida. Lo lamento mucho Antonio, pero es necesario para su salud y para mantenerse en vida.

Tómelo con calma, tiene 2 meses para pensarselo, pero piense en usted y en su familia.

Mucha fuerza Antonio, cualquier duda no dude en comunicarse conmigo por aquí, o llamar al hospital La Fe para más información.

Cordialmente su doctor.

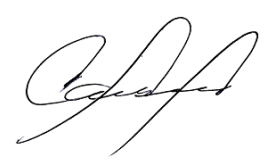

4KarlaBLEE (A1)

En este caso la actividad es inadecuada porque la alumna emplea demasiadas estrategias de atenuación para la situación comunicativa concreta que se le ha presentado. Como ya hemos explicado anteriormente, los comportamientos hipercorteses, también pueden resultar inadecuados (y por tanto, perjudiciales para la relación interpersonal entre los hablantes), ya que una cortesía exagerada puede mostrar una aplicación pragmática fallida (Bernal, 2007: 76). En este caso (ejemplo 4) la alumna, además de invertir mucho espacio para introducir el mensaje que quiere trasmitir, muestra una preocupación excesiva por el paciente y se disculpa en repetidas ocasiones por lo sucedido. De esta forma está evidenciando que ella, como doctora, considera que se trata de un problema grave, algo que puede hacer que la preocupación en el paciente crezca, sin conseguir así el objetivo comunicativo que perseguía a través del mensaje.

\section{PROPUESTA DIDÁCTICA}

Los resultados han mostrado que en más del 40\% de los casos los estudiantes no han sabido adecuarse a las situaciones comunicativas concretas que se les han presentado por no emplear las tácticas atenuantes necesarias y apropiadas. Esto evidencia que la creación de una propuesta didáctica basada en la atenuación les ayudaría a desarrollar de forma significativa la competencia pragmática. Por otra parte, los resultados también han revelado que los alumnos se adecuan mejor pragmáticamente a las situaciones comunicativas a las que están más expuestos en su día a día. En ese sentido, debemos dedicar más atención en el aula a aquellas con las que los alumnos no están tan familiarizados, esto es, las situaciones comunicativas más formales.

En este sentido, hemos diseñado una propuesta didáctica integrada dentro de la asignatura Lengua Castellana y Literatura y dirigida al alumnado de $4^{\circ}$ de la ESO, cuyo objetivo es no solo trabajar y mejorar la competencia pragmática de los alumnos, cubriendo las principales necesidades y carencias observadas en el análisis del corpus, sino también aproximarnos al uso real de la lengua, proponiendo la atenuación como mecanismo para formar alumnos más competentes en los intercambios comunicativos de su día a día. 
La propuesta didáctica se ha dividido en tres sesiones de 90 minutos en las que se han trabajado aspectos de la atenuación, así como expresiones y usos lingüísticos. Estas sesiones se conciben como bloques de trabajo en torno a unos objetivos didácticos concretos, dentro del tema de la atenuación, y no como sesiones de una clase de la asignatura, pues cada sesión abarcaría más de una clase. El orden establecido ha sido de más fácil a más difícil, introduciendo en cada sesión algún elemento nuevo y remitiendo siempre a los aspectos ya vistos, de forma que los tengan que poner en uso.

\subsection{Metodología y objetivos de la propuesta didáctica}

Para diseñar y elaborar las distintas actividades que conforman la propuesta, se ha seguido un enfoque comunicativo en el que se ha favorecido en los estudiantes la necesidad de comunicarse (tanto oralmente como por escrito) para poder resolver las situaciones y problemas planteados. Como explica Viso (2010: 137), el enfoque comunicativo consiste en darles: «datos concretos para reflexionar, analizar y discutir en grupo las posibles salidas que se pueden encontrar a cierto problema; no ofrece soluciones al alumno sino que le permite que se entregue para generarlas él mismo». En concreto, gran parte de la propuesta didáctica sigue el enfoque por tareas, ya que: «Uno de los modos privilegiados de favorecer el desarrollo de las competencias, es decir, de demostrar esas capacidades adquiridas, es el aprendizaje a través de tareas» (Viso, 2010: 140). La forma más directa de demostrar una buena adquisición de todo lo estudiado es la tarea final, en la que los alumnos tienen que utilizar y poner en práctica todo los conocimientos y, especialmente, todas las habilidades que han ido aprendiendo.

Respecto a la agrupación de los alumnos, varia a lo largo de las sesiones dependiendo del tipo de actividad. En algunas actividades se trabaja de forma individual y en otras se establecen agrupaciones que van desde las parejas o grupos reducidos (de 3 a 5 personas), hasta grupos grandes en los que participa toda la clase. No obstante, las agrupaciones más frecuentes para trabajar son las de los grupos reducidos, ya que son una garantía de que todos los estudiantes participan y se ayudan transmitiéndose conocimientos y habilidades mutuamente, evitando así la individualización de estos.

Por otro lado, a lo largo de las tres sesiones se realizan actividades en las que se trabajan las cuatro destrezas básicas (expresión oral, expresión escrita, comprensión oral y comprensión escrita) junto con las dos destrezas que posteriormente incorporó el MCER (2001): la interacción oral y la interacción escrita. A través de ellas los estudiantes actúan conjuntamente para construir una conversación mediante la negociación de significados y pueden practicar la atenuación y sus manifestaciones lingüísticas a través del diálogo para encontrar una solución al problema planteado previamente. Así, estas dos destrezas se convierten en el medio apropiado para conseguir los fines comunicativos que buscan, para trabajar las relaciones sociales y para adecuarse a las distintas relaciones interpersonales.

Los objetivos didácticos generales de esta propuesta didáctica basada en el mecanismo de la atenuación son los siguientes:

- Comprender que para la buena adquisición de la competencia comunicativa no basta con la corrección lingüística.

- Saber qué es la atenuación y entender que puede ayudarles a mejorar su competencia pragmática y su eficiencia comunicativa.

- Conocer qué es y en qué consiste la fuerza ilocutiva de un acto comunicativo.

- Conocer y saber identificar los elementos de las situaciones comunicativas que se 
les presenten en su día a día.

- Saber utilizar las expresiones atenuantes adecuadas a cada situación comunicativa según los elementos que la integran.

- Ser conscientes de que un uso excesivo de tácticas atenuantes puede obstaculizar la comunicación (overpoliteness).

- Saber resolver eficientemente cualquier situación comunicativa que se les presente en su día a día, tanto de la oralidad como de la escritura.

- Utilizar la lengua para expresarse de forma coherente y adecuada en contextos distintos de la actividad social y cultural.

\subsection{Descripción de la actividad}

A continuación, se presenta una tabla (Tabla 5) en la que se desglosan, más detalladamente, las actividades que conforman cada una de las sesiones:

\section{SESIÓN DESARROLO}

\section{Presentación e introducción}

Presentación de la propuesta didáctica e introducción teórica al mecanismo pragmático de la atenuación.

1

\section{TEMPORALIZACIÓN}

20 minutos

30 minutos

Actividad 1: Y la atenuación ¿para qué sirve? Explicación del concepto de "fuerza ilocutiva" o "fin comunicativo". Para ello se muestra una conversación y se plantean preguntas sobre esta para que, de forma deductiva e individualmente, entiendan que para lograr el fin comunicativo no es suficiente con decir el enunciado final, es necesario ser cortés, educado y guardar respeto hacia el otro. Es aquí donde entra en juego la atenuación. Actividad 2: Dime qué quieres, pero dímelo bien

Explicación y puesta en práctica de la función comunicativa de la petición a través de la adquisición de forma deductiva de expresiones lingüísticas y gramaticales (atenuantes) para hacer un buen uso de esta. Por parejas, tienen que ponerse de acuerdo para redactar una serie de peticiones de forma que no dañen la imagen de su interlocutor y que consigan su objetivo comunicativo. Para ello les será muy útil emplear tácticas lingüísticas de atenuación.

Actividad 3: Emoticonízate

En el caso de algunos géneros escritos, hay un elemento que también puede funcionar como atenuante y puede ayudar notablemente a la eficiencia comunicativa: el emoticono. Esta actividad consistirá en que los estudiantes completen las peticiones redactadas en la actividad 2 con los emoticonos que consideren que pueden ayudarles a alcanzar de forma más fácil su objetivo comunicativo. 
Explicación y puesta en práctica de dos funciones comunicativas: la de transmitir noticias delicadas y la de expresar desacuerdo, a través de la adquisición, de forma deductiva, de expresiones lingüísticas y gramaticales (atenuantes) adecuadas para ello. A partir de la proyección de una de las escenas de la película Up in the Air, en la que Ryan (George Clooney) y Natalie (Anna Kendrick) despiden a los empleados de una empresa, se les pide que, en grupos reducidos:

Escriban las expresiones o palabras atenuantes que creen que utilizan tanto Ryan como Natalie para despedir a los empleados

Escriban las expresiones o palabras atenuantes que los empleados utilizan para mostrar su desacuerdo con la noticia que se les está transmitiendo.

Después de haber anotado todos las expresiones que creen que son atenuantes, se procederá conjuntamente a la elaboración de un listado de mecanismos atenuantes para expresar adecuadamente las dos funciones comunicativas señaladas: la de transmitir una noticia delicada y la de expresar desacuerdo.

Actividad 5: Yo creo que...

Explicación y puesta en práctica de la función 25 minutos comunicativa de dar la opinión a través de la adquisición de forma deductiva de expresiones lingüísticas y gramaticales (atenuantes) para hacer un buen uso de esta. Por grupos reducidos, tienen que detectar las expresiones atenuantes de una conversación entre dos amigas en la que una de ellas opina sobre el novio de la otra. Cada grupo expondrá las tácticas con ejemplos que ha encontrado en la conversación y, entre todos y con la ayuda de las retroalimentaciones del profesor, se confeccionará la lista de tácticas atenuantes que sirven para expresar opiniones.

Actividad 6: jVaya situación!

Identificación y comparación de los elementos de las situaciones comunicativas de las actividades 4 y 5. Para ello, contestan en pequeños grupos a una serie de preguntas que les guían en la identificación de dichos elementos situacionales. Finalmente, se procede a la elaboración de mapas conceptuales que incluyan los elementos de ambas situaciones, de forma que vean claramente y comparen de forma visual ambas.

Actividad 7: Reflexionando

15 minutos

Reflexión y puesta en común para extraer las principales conclusiones después de haber realizado todas las actividades. También se hace referencia a la overpoliteness, pues un uso excesivo de tácticas atenuantes también puede obstaculizar la comunicación. Tarea final dividida en dos partes: en la primera se trabajan situaciones comunicativas pertenecientes al registro escrito y en la segunda, situaciones 
Redacción, de forma individual, de una de las diez situaciones comunicativas propias del registro escrito propuestas. En cada una de ellas se trabaja un género escrito concreto y una función comunicativa distinta. Para guiarlos en la realización de la tarea, se les pide que, antes de ponerse a redactar, identifiquen el objetivo o la fuerza ilocutiva que persiguen a través del mensaje y que tengan claro cuáles son los elementos de la situación comunicativa presentada para poder adecuarse a ella. Servirá como retroalimentación un dosier que incluirá todas las situaciones comunicativas corregidas, de forma que los estudiantes dispongan de diversos ejemplos de diferentes situaciones que pueden presentárseles en su día a día, además de saber así la estructura y la forma de redactar cada uno de los distintos géneros.

$\frac{\text { Tarea final (Parte II): Crisis de pareja }}{\text { Representación, por parejas, de una situación }}$ comunicativa propia de la oralidad. Se hace entrega a cada estudiante de una situación que lleva el nombre de un personaje de la historia de la literatura universal que es la pareja de otro. Así, para poder empezar la tarea, tienen que buscar a su respectiva pareja literaria y una vez la hayan encontrado, tienen que adivinar a qué obra literaria pertenecen estos personajes. De la misma forma que en la primera parte de la tarea final, tienen que reconocer previamente cuál es su propósito en la comunicación y cuáles son los elementos de la situación comunicativa que se les presenta. Una vez tengan claros estos aspectos, se procede a la preparación y al desarrollo de la situación comunicativa, para lo cual disponen de vestuario y de elementos decorativos con los que pueden darle verosimilitud a su representación. Así, se cierra la propuesta didáctica con una tarea amena, dinámica y lúdica en la que todos los alumnos pueden aprender cómo afrontar distintas situaciones comunicativas de la oralidad.

Tabla 5. Descripción de las actividades propuestas para trabajar la competencia pragmática a través de la atenuación

\subsection{Evaluación}

Respecto a la evaluación, en cada una de las sesiones hay unos instrumentos a evaluar y cada una pondera de forma diferente, dependiendo de las actividades que incluya. En la Tabla 6 se detallan todos los aspectos referentes al sistema evaluativo adoptado a lo largo de la propuesta didáctica:

\section{SESIONES}

1

\section{PORCENTAJES E INSTRUMENTOS DE EVALUACIÓN}

Esta sesión representa el 25\% de la nota total de la propuesta. En ella, se evalúan, por una parte, las actividades que los alumnos han realizado a lo largo de la sesión, es decir:

La preguntas que contestan en la actividad 1 y la posterior exposición y argumentación de las respuestas al resto de la clase: $5 \%$

Las tres peticiones que redactan en la actividad 2: 10\%

Los ejemplos aportados en cada una de las tácticas de la actividad 2 y la posterior exposición de estas: 5\% 


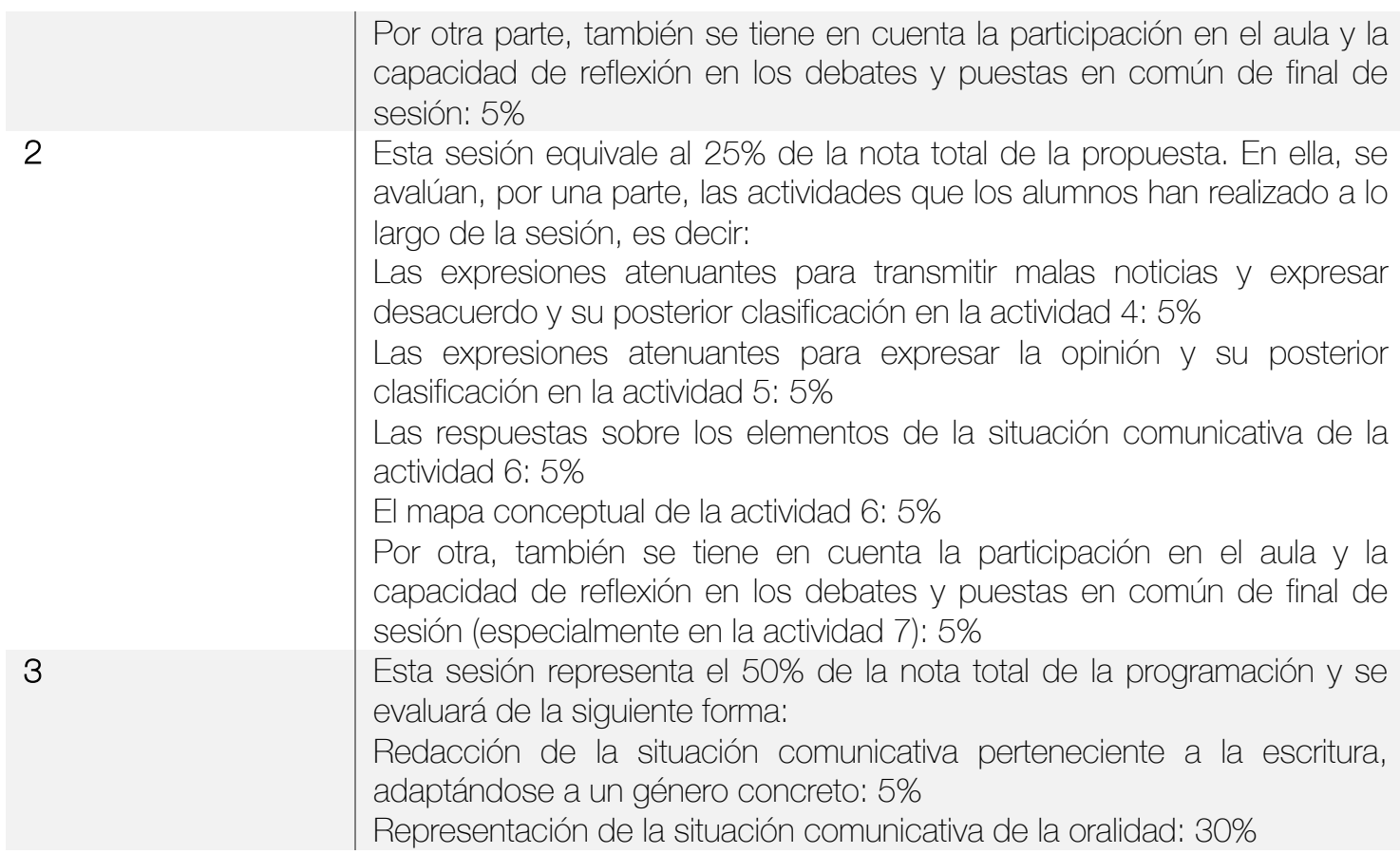

Tabla 6. Sistema de evaluación de la propuesta didáctica

Finalmente, se plantea una propuesta de autoevaluación que sirve, por un lado, para que los alumnos comprueben si han adquirido o no lo estudiado en clase durante las sesiones y, por otro, para que el docente descubra el nivel de adquisición de los alumnos, y conozca qué aspectos están interiorizados y qué aspectos necesitan reforzarse y trabajarse más. Además, la autoevaluación que realizan los alumnos sirve también para comprobar si la forma de plantear la propuesta didáctica ha sido la adecuada o si necesita mejorar.

\section{CONCLUSIONES}

A través de este estudio se ha pretendido reivindicar una mayor atención a la competencia pragmática en los cursos de lengua en el sistema educativo de la secundaria. Para ello, se ha propuesto la atenuación lingüística, un mecanismo de naturaleza pragmática que puede ayudar a mejorar de forma notable la eficiencia comunicativa y las relaciones interpersonales. Así, se ha evaluado la adecuación en el uso de este mecanismo en estudiantes de $4^{\circ}$ de la ESO a través de un estudio empírico en el que se ha recogido un corpus escrito de 88 actividades en las que se presentan situaciones comunicativas concretas que los estudiantes tienen que resolver. Para efectuar la evaluación de la competencia pragmática se ha diseñado un sistema basado en el mayor o menor uso de la atenuación en el que se establecen tres niveles de adecuación pragmática: adecuado, inadecuado por defecto e inadecuado por exceso (basado parcialmente en Secchi, 2017).

Los resultados de dicho análisis son los siguientes: en la mayoría de los casos (59,09\%) los alumnos han sabido adecuarse a la situación comunicativa propuesta, haciendo un buen uso de la competencia pragmática, para lo cual se han ayudado y apoyado en el uso de las estrategias de atenuación apropiadas y necesarias en cada caso. Sin embargo, ha de hacerse notar que incluso las actividades que han sido consideradas adecuadas, podrían mejorarse a través del empleo de la atenuación, lo que les permitiría desempeñar un mayor uso retórico de la lengua y asegurarse que los destinatarios reciben los mensajes con mayor aceptación. Asimismo, más del $40 \%$ de actividades han sido inadecuadas, ya sea por defecto $(35,22 \%)$ o por exceso $(5,68 \%)$. Este resultado pone de manifiesto la 
necesidad de diseñar una propuesta didáctica basada en la implementación de la atenuación que les permita desarrollar una buena competencia pragmática y ser eficientes comunicativamente.

Por último, conviene señalar que los resultados obtenidos en este análisis no son representativos para toda la lengua, puesto que nos hemos centrado solo en una muestra de corpus, y en dos géneros específicos, como son el correo electrónico y el WhatsApp. En futuros estudios habría que ampliar la muestra y realizar estudios transgenéricos, observando qué ocurre en los diferentes géneros discursivos de la lengua y en otras situaciones comunicativas. Somos conscientes de esto y este estudio solo constituye un inicio en la investigación de la estrategia lingüística de la atenuación en los estudiantes de secundaria y de la competencia pragmática de estos.

\section{REFERENCIAS BIBLIOGRÁFICAS}

BACHMAN, Lyle (1990): Fundamental Considerations in Language Testing, Oxford, Oxford University Press.

BERnAL, María (2007): Categorización sociopragmática de la cortesía y de la descortesía (tesis doctoral), Estocolmo, Universidad de Estocolmo.

BIALYSTOK, Ellen (1993): «Symbolic Representation and Attentional Control in Pragmatic Competence», en Kasper, G. y Blum-Kulka, S., eds., Interlanguage Pragmatics, Nueva York, Oxford, 43-57.

Boletín Oficial del Estado. Ley Orgánica 2/2006, de 3 de mayo, de Educación.

BRIZ, Antonio (1998): El español coloquial en la conversación, Barcelona, Ariel.

Briz, Antonio y Marta AlbeldA (2013): «Una propuesta teórica y metodológica para el análisis de la atenuación lingüística en español y portugués. La base de un proyecto en común (ES.POR.ATENUACIÓN)», Onomazein, 28, 288-319.

BULNES, Mar (1993): Materiales Didácticos. Lengua Castellana y Literatura, Madrid, Ministerio de Educación y Ciencia.

CANALE, Michael (1983): «From communicative competence to communicative language pedagogy", en Richards, J. C. y Schmidt, R. W., eds., Language and Communication, Londres, Longman, 2-27.

CANALE, Michael y Merrill SWAIN (1980): «Theoretical basis of communicative approaches to second language teaching and testing», Applied Linguistics, 1, 1-47.

CELCE-MuRCIA, Marianne, Zoltan DöRnYEl y Sarah THURRELL (1995): «Communicative competence: A Pedagogically motivated framework with content specifications", Issues in Applied Linguistics, 6, 5-35.

CONSEJO DE EUROPA (2001): Marco común europeo de referencia para las lenguas: aprendizaje, enseñanza, evaluación, Madrid, MECD y Anaya.
FERNÁNDEZ, Jesús (1998): «Teoría pragmática y análisis lingüístico en secundaria», CAUCE, Revista de Filología y su Didáctica, 20-21, 557591.

GonZÁLEZ, Aurelio et alii (2011): El aprendizaje por competencias en la educación obligatoria, Valencia, Brief.

GonZÁlEZ, Virginia y Amparo García (2017): «Atenuación e intensificación: estrategias pragmáticas del rechazo en respuestas a invitaciones en redes sociales en línea», en Albelda, M. y Mihatsch, W., eds., Atenuación e intensificación en géneros discursivos, Madrid, Iberoamericana Vervuert, 187-203.

HYMES, Dell (1972): «On communicative competence», en Pride, J. B. y Holmes, J., eds., Sociolinguistics, Harmondsworth, Penguin, 269-285.

LOCHER, Miriam y Richard J. WATTS (2005): "Politeness theory and relational work», Journal of Politeness Research, 1, 9-33.

LOMAS, Carlos (1994): «La educación lingüística y literaria», Textos de Didáctica de la Lengua y la Literatura, 1, 8-17.

LOMAS, Carlos (1997): "La educación lingüística y el limbo de los justos», Signos. Teoría y práctica de la educación, 22, 62-63.

LOMAS, Carlos, Andrés OsORO y Amparo Tusón (1993): Ciencias del Lenguaje, competencia comunicativa y enseñanza de la lengua, Barcelona, Paidós.

MedinA, Antonio (2009): Formación y Desarrollo de las competencias básicas, Madrid, Editorial Universitas, S.A.

SECCHI, Daniel (2017): «La enseñanza de la atenuación en E/LE a partir del análisis de un corpus real», Foro de Profesores de E/LE, 13, 247-257.

VAN EK, Jan (1986): Objectives for Foreign Language Learning (Vol. I), Estrasburgo, Council of Europe.

VISO, José Ramiro (2010): Qué son las competencias (Vol. I), Madrid, EOS. 
Revisión de la atenuación como expresión de la competencia pragmática | À. Magraner 